\title{
AC 2007-2906: INNOVATIVE STRATEGIES FOR TEACHING CONSTRUCTION FUNDAMENTALS: IMPLEMENTING DESIGN-BUILD PROJECT DELIVERY PROCEDURES INTO A COURSE IN COST ESTIMATING
}

\section{David Cottrell, University of North Carolina-Charlotte}

DR. DAVID S. COTTRELL is an Assistant Professor in the Department of Engineering Technology, University of North Carolina at Charlotte. He graduated from the United States Military Academy in 1978 and retired in 2000 after more than 22 years of service with the US Army Corps of Engineers. Studies at Texas A\&M University resulted in an MS Degree in Civil Engineering in 1987 and a PhD in 1995. He is a registered Professional Engineer and has taught courses in statics, dynamics, mechanics of materials, graphic communications, engineering economy, and construction planning, scheduling, estimating, and management. 


\section{Innovative Strategies for Teaching Construction Fundamentals: Implementing Design-Build Project Delivery Procedures into a Course in Cost Estimating}

\section{Introduction}

This paper describes the integration of design-build projects during the Fall Semester, 2006, into a cost estimating course in the Department of Engineering Technology at the University of North Carolina at Charlotte. Design-build is a project delivery system with an enviable record of bringing capital facilities on-line rapidly often with reduced overall project costs. ${ }^{1,2}$ Nevertheless, the procedure tends to be associated with higher risk as it radically short-cuts the traditional design-bid-build protocol where the contractor routinely receives an essentially completed design package prior to the development of a detailed estimate. Tradition methods allow a deliberate, independent development of work package quantities, durations, crew development, and assignment of costs. This paper examines the creation of a block of instruction in a conventional cost estimating course that addresses the salient characteristics of the alternative design-build project delivery particularly as it impacts the creation of project cost estimate including:

- The collaborative relationship between owners, designers, and constructors from initial project development through construction.

- The use of a general scope of work in the absence of completed designs to support creation of a conceptual estimate that determines total project costs based on:

- Features that can be readily priced with confidence;

- Alternatives for features that have yet to be adequately defined.

This paper examines ETCE 3281, a course in cost estimating, primarily for $3^{\text {rd }}$ year students pursuing a Construction Management or Civil Engineering Technology Degree. Course content is conventional with the study of industry wide standard techniques and practices for estimating costs of construction activities. Students typically prepare quantity surveys and estimate labor and equipment requirements for well defined work packages resulting from often finitely developed contract documents, specifications, and drawings. This paper examines the implementation of practical exercises and project scenarios that create a realistic project atmosphere simulating a successful design-build environment complete with partial designs and imperfect information. Students developed conceptual estimates in an iterative fashion in consultation with the "owner" and with the "designer" as they continued to develop and refine project features. This paper will present:

- The project documents employed in the practical exercise to develop the design-build scenario; 
- The techniques and resources employed by the students to prepare cost estimates;

- Assessment data from the students pertaining to their perception of designbuild as a viable project delivery method and as it compares to traditional design-bid-build detailed estimating protocols.

This paper will document the utility of implementing current, relevant industry practices into convention courses.

\section{Course Description}

This paper examines ETCE 3281, a course in construction cost estimating primarily for $3^{\text {rd }}$ year students pursuing a degree in either Construction Management or Civil Engineering Technology. ETCE 3281 provides the student with a working knowledge of the concepts, terminology, and methods associated with estimating the costs of construction activities. Students who successfully complete this course will be able to visualize the construction process pertinent to the selected project delivery, to understand the way work is to be performed, and the time required based upon a set of drawings and specifications. The resulting estimate is the basis for the bid which may or may not be good enough to win the job. No two projects are exactly alike, yet the contractor is frequently called upon to set a fixed price for the work to be performed.

\section{Student Projects}

It is often held that the accuracy of any estimate will depend on the amount of information known for a project. ${ }^{3}$ One of the significant teaching points of this block of instruction was to allow the students to appreciate the accepted techniques within the industry for developing a conceptual estimate based on limited information. Conceptual estimates of this nature providing the owner with an approximate cost to evaluate the project economic feasibility. Nevertheless, applying these techniques in the DesignBuild arena works primarily for the contractor to serve as a basis for entering into a contract for design and constructive services. Almost without question, the contractor must rely on historical data pertaining to past performances with projects of similar scope. Based on the past accomplishments, he formulates a methodology that will allow quantifiable projects to predict a future cost of a desired facility. The students of ETCE 3281 employed the following techniques to develop their estimates. Since this is based on historical data, estimates must often be adjusted for a variety of factors to account for variations in scope, budget, and/or schedule issues. Adjustments for time, location, size, and inflation are not uncommon and may prove valuable to create credibility for the estimate. The accuracy must be strictly assessed and appropriate contingencies be added to account for associated risk. In each case, these estimates are relatively straightforward and rapid in their development of a projected cost estimate and served as a good prelude for the detailed estimates coming later in the cost that build on essentially completed design drawings, specifications, and contract/bid documents. 
Cost Capacity Curves: This technique represents a graphical approach and provides a rapid determination of a future cost. This approach can be used for a variety of facilities such as process units, systems, and services. Typically, these "cost vs. capacity" curves are prepared by specialists who develop, maintain, and update the curves based on final, return cost data on completed jobs. The problem presented to the students of ETCE 3281 dealt specifically with estimating the cost for a refinery project with a particular process unit and an expected capacity.

Capacity Ratios Raised to an Exponent: Also compatible with the process industry, this technique is more quantifiable that the graphical methods presented earlier. In this approach, the costs and the capacities of a known completed facility are related by a mathematical expression shown below:



Estimators correlate historical data from completed records and employs standard statistical procedures to develop the exponent $-\mathrm{X}$ - that is the key to the effectiveness of the process. The exponent will normally range between 0.55 and 0.88 depending on the type of facility. For application, the students were provided information pertaining to a previous process unit with a known capacity, cost, and analytical results yielding a certain capacity ratio exponent from historical records. Students developed an estimated cost of a similar process unit with an expected capacity.

Weighted Unit Cost: The estimator analyzes previously completed projects to determine a productive statistic regarding the historical unit cost which is then used to forecast future performance on a proposed project. Procedures employ the following equation in order to develop a "weighted" unit cost:

$$
\text { Unit Cost }=\frac{A+4 B+C}{6}
$$

Where $\quad \begin{aligned} & \mathrm{A}=\text { minimum unit cost of previous projects } \\ & \mathrm{B}=\text { average unit cost of previous projects } \\ & \mathrm{C}=\text { maximum unit cost of previous projects }\end{aligned}$

This Unit Cost in \$ per unit can be summarily used as a factor for estimating the cost of a new facility of known size. As a practical demonstration, the students were provided historical data for a number of previously completed projects which provided them the means for completing a cost analysis and enabled them to project the estimated cost of a particular project of known size. 


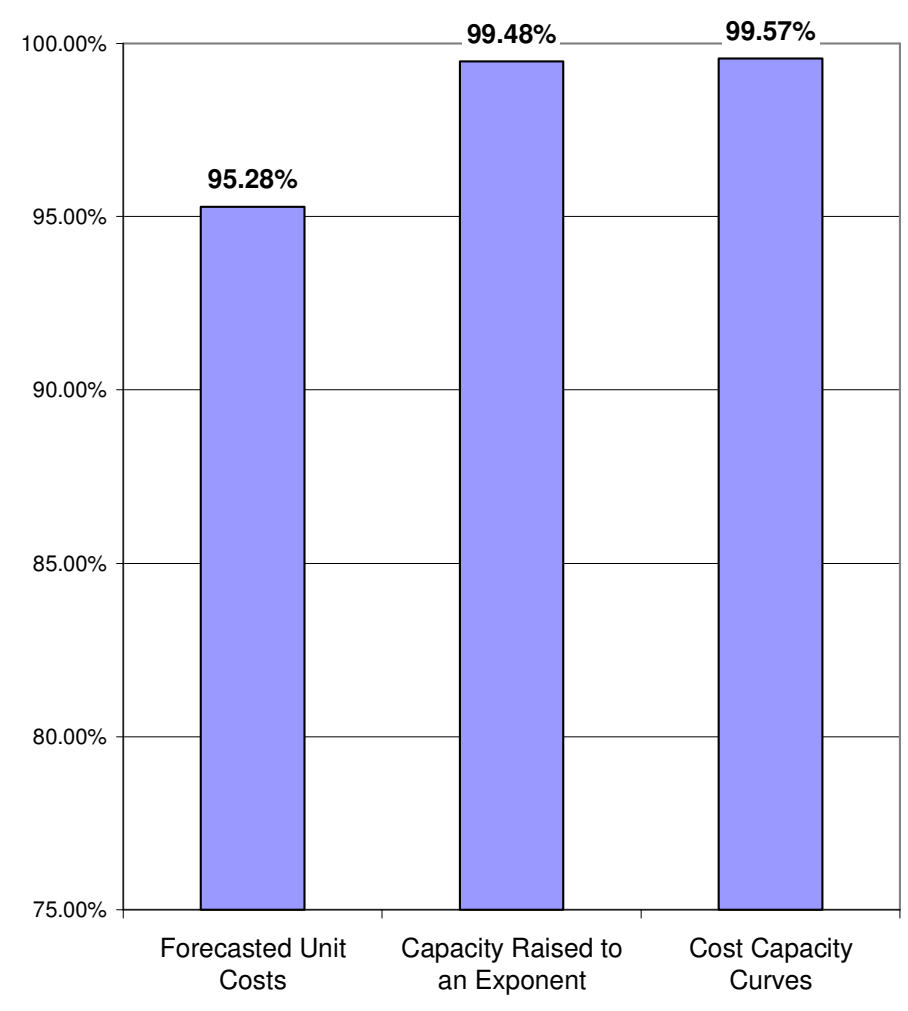

Figure 1: Student Performance of Graded Conceptual Estimating Exercises

\section{Student Performance, Feedback, and Conclusion}

As can be seen from the figure above, student performance was superb. They clearly mastered the mechanics of these relatives straight forward application of techniques for developing estimates when information about the proposed project is lacking. Designbuild projects can have as much as $25 \%$ of the design completed when the contractor begins to develop his estimate of the cost. Student surveys concerning the estimating process in the face of little of no information indicated that initially the students were skeptical that any estimate could be set with the given conditions. However, afterwards, they were highly confident in their ability to envision a baseline cost and to apply appropriate adjustment factors to account for any variations between the historical and future projects' scope, budget, or schedule. This exercise indicates the benefit of taking advantage of a target of opportunity to demonstrate that the students - and, of course, constructors - can sometimes actually do quite a lot with just a little, especially when it is necessary to perform cost estimating with an incomplete design. Clearly, although certainty and confidence correlate closely with design completeness, methodologies do exist which can provide early in the construction process a credible glimpse of the cost of the venture. 


\section{Bibliography:}

1. Gransberg, Douglas D., James E. Koch, and Keith Molenaar, Preparing for Design Build Projects, American Society of Civil Engineers, Reston, Virginia, 2006.

2. Levy, Sidney M., Design Build Project Delivery, McGraw-Hill, New York, New York, 2006.

3. Peurifoy, Robert L. and Garold D. Oberlender, Estimating Construction Costs, McGraw-Hill, $5^{\text {th }}$ Edition, New York, New York, 2002. 